\title{
Biorthogonal Wavelet Based Algebraic Multigrid Preconditioners for Large Sparse Linear Systems
}

\author{
A. Padmanabha Reddy, Nagendrappa M. Bujurke* \\ Department of Mathematics, Karnatak University, Dharwad, India \\ E-mail: "bujurke@yahoo.com
}

Received June 24, 2011; revised July 23, 2011; accepted August 1, 2011

\begin{abstract}
In this article algebraic multigrid as preconditioners are designed, with biorthogonal wavelets, as intergrid operators for the Krylov subspace iterative methods. Construction of hierarchy of matrices in algebraic multigrid context is based on lowpass filter version of Wavelet Transform. The robustness and efficiency of this new approach is tested by applying it to large sparse, unsymmetric and ill-conditioned matrices from Tim Davis collection of sparse matrices. Proposed preconditioners have potential in reducing cputime, operator complexity and storage space of algebraic multigrid V-cycle and meet the desired accuracy of solution compared with that of orthogonal wavelets.
\end{abstract}

Keywords: Algebraic Multigrid, Preconditioner, Wavelet Transform, Sparse Matrix, Krylov Subspace Iterative Methods

\section{Introduction}

The linear system of algebraic equations

$$
A x=b
$$

where $A$ is $n \times n$ non-singular matrix and $b$ is vector of size $n$ arise while discretising various equations using finite difference, finite element and domain decomposition schemes etc.

One of the useful schemes to solve (1.1) is multigrid. For multigrid (geometric/algebraic), if one has used the proper smoother, restriction and prolongation operators, then the multigrid algorithm will require so few cycles to reach the level of truncation error. But unfortunately such operators are not always known for all problems. In such cases, acceleration of Krylov subspace iterative methods will help. Equivalently, one can also consider multigrid as a preocnditioner for one of the Krylov subspace iterative methods [1].

In classical schemes, designing/constructing a suitable preconditioner for unstructured matrix $\boldsymbol{A}$-arising from nonsmooth region or mesh free type problems is an impossible/tough task. Furthermore, Algebraic multigrid-a general purpose purely algebraic scheme which relays only on the elements of $A$ and works as universal pre- conditioner heralds a cutting edge of current research on preconditioners.

Orthogonal (Daubechies) wavelet based preconditioners are developed for various types of large sparse matrices [2-4]. Kumar and Mehra [2] developed matrix splitting based preconditioners, where as in [3] and [4] wavelet based algebraic multigrid as preconditioners for Krylov subspace iterative methods are developed. In these articles authors have shown that wavelet based algorithms are efficient and robust compared with that of classical schemes such as matrix splitting, algebraic multigrid and incomplete LU factorization(ilu) based preconditioners for Krylov subspace iterative methods. These studies motivate us to develop biorthogonal wavelet based preconditioners for Krylov subspace iterative methods.

\section{Discrete Biorthogonal Wavelet Transform}

For a given $p, \tilde{p} \in \mathbb{N}$, such that $p+\tilde{p}$ is even, there exists compactly supported biorthogonal spline wavelets of order $p, \tilde{p}$ called Cohen Daubechies Feauveau wavelets $(\mathrm{CDF}(p, \tilde{p}))$ which form biorthogonal bases for $L_{2}(R)$ [5]. Let $S$ be a vector (signal) of length $n$, its one level biorthogonal wavelet transform is given by Ws . For $\operatorname{CDF}(2,2)$, its wavelet transform matrix is 


$$
\begin{aligned}
& W= \\
& \left(\begin{array}{ccccccccc}
\tilde{h}_{0} & 2 \tilde{h}_{1} & & & & & & & \\
& \tilde{h}_{-1} & \tilde{h}_{0} & \tilde{h}_{1} & & & & & \\
& \vdots & & \ddots & & \vdots & & & \\
& & & & & & \tilde{h}_{-1} & \tilde{h}_{0} & \tilde{h}_{1} \\
\tilde{g}_{0} & \tilde{g}_{-1}+\tilde{g}_{1} & \tilde{g}_{2} & \tilde{g}_{3} & & & & & \\
& \tilde{g}_{-1} & \tilde{g}_{0} & \tilde{g}_{1} & \tilde{g}_{2} & \tilde{g}_{3} & & & \\
& & \vdots & & \ddots & & \vdots & & \\
& & & & & & 2 \tilde{g}_{-1} & 2 \tilde{g}_{0} & \tilde{g}_{1}
\end{array}\right)_{n \times n}
\end{aligned}
$$

Matrix $\boldsymbol{W}$ contains first $n / 2$ rows which are lowpass filter coefficients and remaining $n / 2$ rows are highpass filter coefficients. $W$ can be derived for various $p$ and $\tilde{p}$ by symmetric extension of filters [6] and $n$ is any natural number [3]. Here first half of $W s$ is a representation of $s$ at a lower resolution level. This representation has to preserve average, first moment and so on of the original signal $s$. Preservation of these moments depends on the accuracy of the scaling function used [6,7].

To create wavelets on higher dimensional domains, one of the approaches is to perform the wavelet transform independently for each dimension. For two dimensional cases, let $A$ be a $n \times n$ matrix then its wavelet transform is

$$
\tilde{A}=W A W^{T}
$$

Here $\tilde{A}$ contains four types of coefficients/subbands: LL-lowpass in both the horizontal and vertical directions (approximation/average coefficients), LH-lowpass in the vertical, highpass in the horizontal direction, $\mathrm{HL}$ and $\mathrm{HH}$ (detail coefficients). When iterated on the approximation coefficients, the result is multiresolution decomposition as shown in the Figure 1.

This LL subband contains lowpass information, which is essentially a low resolution and represents a coarser version of the original matrix $A$ very much. This concept has been used in obtaining the hierarchy of matrices in the algebraic multigrid (AMG) context.

\section{Wavelet-Based AMG}

Wavelet Transform will be used to generate the hierarchy of matrices in the AMG method. As a result, the coarsening process is eliminated and the setup phase is summarised by a simple choice of the lowpass filters and assembly of the intergrid (restriction and interpolation) operators. In the algebraic multigrid context it is not necessary to rebuild the matrix. Thus, the detail coefficients produced by wavelet transform can be discarded $[3,4]$. In other words, the transformation must produce only one approximation of the original matrix in each decomposition level. Therefore, only the lowpass filters that capture

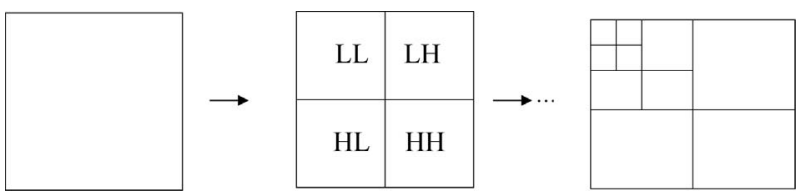

Figure 1. Two-dimensional wavelet transform: iteration on the $L L$ subbands (average coefficients).

the approximation are used in the construction of the matrix $\boldsymbol{W}$ and it is used as a restriction operator for the wavelet based AMG. Using $\operatorname{CDF}(2,2)$ lowpass filters, the restriction operator takes the following form:

$$
R=\left(\begin{array}{ccccccc}
\tilde{h}_{0} & 2 \tilde{h}_{1} & & & & & \\
& \tilde{h}_{-1} & \tilde{h}_{0} & \tilde{h}_{1} & & & \\
& \vdots & \ddots & \ddots & \vdots & & \\
& & & & \tilde{h}_{-1} & \tilde{h}_{0} & \tilde{h}_{1}
\end{array}\right)_{n / 2 \times n}
$$

The prolongation operator $\left(P=R^{T}\right)$ and the next matrix of hierarchy, in the corresponding level, are defined by $R A R^{T}$, where $R^{T}$ is transpose of $R$.

\section{Cost of Orthogonal and Biorthogonal Wavelet Transforms}

Let $s$ be a vector of length $n$ and let $\tilde{p}$ an accuracy of orthogonal (Daubechies) and biorthogoanal(CDF) scaling functions used. One of the lowpass filter lengths of CDF wavelets is $\tilde{p}+1$, whereas for Daubechies it is $2 \tilde{p}$. Lowpass filtered version of wavelet transform applied to $s$ is of length $n / 2$. Number of operations (multiplication and addition) to obtain this for Daubechies filters is $\tilde{p} n$, where as for CDF filters it is of $(\tilde{p}+1) n / 2$. For large $n$, we can save the number of operations and setup time (performing WT) by using biorthogonal scaling filters compared with that of orthogonal scaling filters. Similar arguments hold for cost of individual wavelet transform in higher dimensions.

Grid complexity and operator complexity are two simple criteria used to provide a posterior cost estimates as defined in [3] for AMG V-cycle. Here grid complexity is same for both families of wavelets. The advantage of reduction of the cost and setup time for biorthogonal wavelet transform results into the reduction of operator complexity compared with that of orthogonal wavelet transform for fixed accuracy of scaling functions. Operator complexity indicates the total storage space required by the hierarchy of matrices over all levels and is generally considered as a good indicator of the expense of the AMG V-cycle.

We have calculated operator complexity and setup time for both families of wavelets for various unsymmetric matrices given in Table 1, from Tim Davis [8] collection of sparse matrices. The number of levels chosen 
Table 1. Operator complexity and setup phase cputime in seconds (number in square bracket) for various matrices.

\begin{tabular}{|c|c|c|c|c|}
\hline Matrix Name and size & Daub-2 & $\operatorname{CDF}(2,2)$ & Daub-3 & $\operatorname{CDF}(3,3)$ \\
\hline $\begin{array}{l}\text { Poisson 2D } \\
{[367 \times 367]}\end{array}$ & $\begin{array}{c}6.65 \\
{[0.0156]}\end{array}$ & $\begin{array}{c}4.62 \\
{[0.0156]}\end{array}$ & $\begin{array}{c}10.02 \\
{[0.0312]}\end{array}$ & $\begin{array}{c}6.59 \\
{[0.0312]}\end{array}$ \\
\hline $\begin{array}{c}\text { Sherman } 4 \\
{[1104 \times 1104]}\end{array}$ & $\begin{array}{c}4.23 \\
{[0.0372]}\end{array}$ & $\begin{array}{c}3.35 \\
{[0.0364]}\end{array}$ & $\begin{array}{c}6.09 \\
{[0.0374]}\end{array}$ & $\begin{array}{c}4.20 \\
{[0.0312]}\end{array}$ \\
\hline $\begin{array}{c}\text { Thermal } \\
{[3456 \times 3456]}\end{array}$ & $\begin{array}{c}2.23 \\
{[0.0936]}\end{array}$ & $\begin{array}{c}1.89 \\
{[0.0728]}\end{array}$ & $\begin{array}{c}2.56 \\
{[0.1248]}\end{array}$ & $\begin{array}{c}2.26 \\
{[0.0988]}\end{array}$ \\
\hline $\begin{array}{c}\text { Airfoil_2D } \\
{[14214 \times 14214]}\end{array}$ & $\begin{array}{c}6.35 \\
{[0.9100]}\end{array}$ & $\begin{array}{c}4.79 \\
{[0.7800]}\end{array}$ & $\begin{array}{c}9.00 \\
{[1.3988]}\end{array}$ & $\begin{array}{c}6.34 \\
{[0.9880]}\end{array}$ \\
\hline $\begin{array}{c}\text { Epb1 } \\
{[14734 \times 14734]}\end{array}$ & $\begin{array}{c}3.08 \\
{[0.6136]}\end{array}$ & $\begin{array}{c}2.53 \\
{[0.5720]}\end{array}$ & $\begin{array}{c}4.25 \\
{[0.8840]}\end{array}$ & $\begin{array}{c}3.08 \\
{[0.7124]}\end{array}$ \\
\hline $\begin{array}{c}\text { Epb2 } \\
{[25228 \times 25228]}\end{array}$ & $\begin{array}{c}3.25 \\
{[1.5756]}\end{array}$ & $\begin{array}{c}2.62 \\
{[1.3468]}\end{array}$ & $\begin{array}{c}4.52 \\
{[2.3192]}\end{array}$ & $\begin{array}{c}3.25 \\
{[1.7316]}\end{array}$ \\
\hline
\end{tabular}

Table 2. Convergence details: Numbers in the columns represent the required number of iterations for the convergence of the respective method and number in square bracket represents the cputime in seconds.

\begin{tabular}{|c|c|c|c|c|}
\hline Matrix Name & Daub-2 & $\mathrm{CDF}(2,2)$ & Daub-3 & $\operatorname{CDF}(3,3)$ \\
\hline Poisson 2D & $\begin{array}{c}12 \\
{[0.2496]}\end{array}$ & $\begin{array}{c}13 \\
{[0.2340]}\end{array}$ & $\begin{array}{c}13 \\
{[0.3120]}\end{array}$ & $\begin{array}{c}13 \\
{[0.2704]}\end{array}$ \\
\hline Sherman4 & $\begin{array}{c}9 \\
{[0.2236]}\end{array}$ & $\begin{array}{c}9 \\
{[0.2288]}\end{array}$ & $\begin{array}{c}8 \\
{[0.2496]}\end{array}$ & $\begin{array}{c}9 \\
{[0.2444]}\end{array}$ \\
\hline Thermal & $\begin{array}{c}4 \\
{[0.5148]}\end{array}$ & $\begin{array}{c}5 \\
{[0.6084]}\end{array}$ & $\begin{array}{c}5 \\
{[0.7176]}\end{array}$ & $\begin{array}{c}5 \\
{[0.7020]}\end{array}$ \\
\hline Airfoil_2D & $\begin{array}{c}80 \\
{[72.9821]}\end{array}$ & $\begin{array}{c}78 \\
{[59.9510]}\end{array}$ & $\begin{array}{c}82 \\
{[103.6990]}\end{array}$ & $\begin{array}{c}75 \\
{[70.8433]}\end{array}$ \\
\hline Epb1 & $\begin{array}{c}129 \\
{[67.7866]}\end{array}$ & $\begin{array}{c}111 \\
{[52.3933]}\end{array}$ & $\begin{array}{c}124 \\
{[87.9266]}\end{array}$ & $\begin{array}{c}92 \\
{[52.6299]}\end{array}$ \\
\hline Epb2 & $\begin{array}{c}49 \\
{[35.3999]}\end{array}$ & $\begin{array}{c}45 \\
{[28.2733]}\end{array}$ & $\begin{array}{c}48 \\
{[46.7166]}\end{array}$ & $\begin{array}{c}41 \\
\text { [31.3933] }\end{array}$ \\
\hline
\end{tabular}

is such that in the coarsest level the dimension is less than 100 , using the expression levels = ceil $(\log 2(n / 15))$, where $n$ is the dimension of the matrix and ceil $(x)$ : rounds $x$ to the nearest integer towards minus infinity.

\section{Numerical Experiments}

To test the efficiency of biorthogonal wavelet based AMG preconditioners and compare with orthogonal wavelet based AMG preconditioners, we have considered the examples given in Table 1. The right hand side of linear system was computed from the solution vector $x$ of all ones (except for Sherman 4 matrix). The initial guess is always $x_{0}=0$ and stopping criteria is relative residual is less than or equal to $10^{-6}$ and the Krylov subspace method adapted is GMRES (20) [1]. Besides these GaussSeidel is used as smoother with number of iterations taken as two. These algorithms are implemented using Matlab-7.5 and Mathematica-7 over machine with Intel Core 2 Duo processor of $1.5 \mathrm{GHz}, 667 \mathrm{MHz}$ FSB and 3 GB RAM corresponding results are shown in Table 2.

Computed results given in Tables $\mathbf{1}$ and $\mathbf{2}$ show that the biorthogonal wavelet based preconditioners developed here, give reduction in operator complexity and require lesser/same number of iterations or cputime. This enables in obtaining solution of required accuracy.

\section{Conclusions}

For a given accuracy of scaling functions, preconditioners designed here have shorter cputime and lower operator complexity leading to reduction of cumulative truncation errors, storage space and improvement of overall accuracy, which are illustrated with examples given in Tables 1 and 2. These two concepts play a crucial role in scientific computing. The preconditioners developed here can also be used for other Krylov subspace iterative methods.

\section{Acknowledgements}

This research work is supported by DST (SR/S4/MS: 281/05), N. M. Bujurke acknowledges the financial support of INSA, New Delhi.

\section{References}

[1] Y. Saad, "Iterative Methods for Sparse Linear Systems," SIAM, Philadelphia, 2003. doi:10.1137/1.9780898718003

[2] B. V. R. Kumar and M. Mehra, "Wavelet Based Preconditioners for Sparse Linear systems," Applied Mathematics and Computation, Vol. 171, No. 1, 2005, pp. 203-224. 
doi:10.1016/j.amc.2005.01.060

[3] F. H. Pereira, S. L. L. Verardi and S. I. Nabeta, “A Wavelet-Based Algebraic Multigrid Preconditioner for Sparse Linear Systems," Applied Mathematics and Computation, Vol. 182, No. 2, 2006, pp. 1098-1107. doi:10.1016/j.amc.2006.04.057

[4] V. M. Garcia, L. Acevedo and A. M. Vidal, "Variants of Algebraic Wavelet Based Multigrid Methods: Applications to Shifted Linear Systems," Applied Mathematics and Computation, Vol. 202, No. 1, 2008, pp. 287-299. doi:10.1016/j.amc.2008.02.015

[5] A. Cohen, I. Daubechies and J. C. Feauveau, "Bior- thogonal Bases of Compactly Supported Wavelets," Communications on Pure and Applied Mathematics, Vol. 45, No. 5, 1992, pp. 485-560. doi:10.1002/cpa.3160450502

[6] F. Keinert, "Wavelets and Multiwavelets," Champan \& Hall/CRC Press, Boca Raton, 2004.

[7] W. Sweldens and P. Schroder, "Building Your Own Wavelets at Home,” In Wavelets in Computer Graphics, ACM SIGGRAPH course notes, 1996, pp. 15-87.

[8] T. Davis, University of Florida Sparse Matrix Collection, NA Digest, 1997.

http://www.cise.ufl.edu/research/sparse/matrices/ 